\title{
Use of common analgesic medications and ovarian cancer survival: results from a pooled analysis in the Ovarian Cancer Association Consortium
}

Suzanne C Dixon ${ }^{\star}, 1,2$, Christina M Nagle ${ }^{1,2}$, Nicolas Wentzensen ${ }^{3}$, Britton Trabert ${ }^{3}$, Alicia Beeghly-Fadiel ${ }^{4}$, Joellen M Schildkraut ${ }^{5,6}$, Kirsten B Moysich7 , Anna deFazio ${ }^{8,9}$, Australian Ovarian Cancer Study Group ${ }^{1,10}$, Harvey A Risch ${ }^{11}$, Mary Anne Rossing ${ }^{12,13}$, Jennifer A Doherty ${ }^{14}$, Kristine G Wicklund ${ }^{12}$, Marc T Goodman ${ }^{15,16}$, Francesmary Modugno ${ }^{17,18,19}$, Roberta B Ness ${ }^{20}$, Robert P Edwards ${ }^{17,18}$, Allan Jensen ${ }^{21}$, Susanne K Kjær ${ }^{21,22}$, Estrid Høgdall ${ }^{21,23}$, Andrew Berchuck ${ }^{24}$, Daniel W Cramer $^{25}$, Kathryn L Terry ${ }^{25,26}$, Elizabeth M Poole ${ }^{27}$, Elisa V Bandera ${ }^{28,29}$, Lisa E Paddock ${ }^{29,30}$, Hoda Anton-Culver ${ }^{31,32}$, Argyrios Ziogas ${ }^{31}$, Usha Menon ${ }^{33}$, Simon A Gayther ${ }^{34}$, Susan J Ramus ${ }^{35,36}$, Aleksandra Gentry-Maharaj ${ }^{33}$, Celeste Leigh Pearce ${ }^{34,37}$, Anna H Wu ${ }^{34}$, Malcolm C Pike ${ }^{38}$ and Penelope $\mathrm{M} \mathrm{Webb}^{1,2}$ on behalf of the Ovarian Cancer Association Consortium

Background: Nonsteroidal anti-inflammatory drugs (NSAIDs) have been associated with improved survival in some cancers, but evidence for ovarian cancer is limited.

Methods: Pooling individual-level data from 12 Ovarian Cancer Association Consortium studies, we evaluated the association between self-reported, pre-diagnosis use of common analgesics and overall/progression-free/disease-specific survival among 7694 women with invasive epithelial ovarian cancer (4273 deaths).

Results: Regular analgesic use (at least once per week) was not associated with overall survival (pooled hazard ratios, pHRs (95\% confidence intervals): aspirin 0.96 (0.88-1.04); non-aspirin NSAIDs 0.97 (0.89-1.05); acetaminophen 1.01 (0.93-1.10)), nor with progression-free/disease-specific survival. There was however a survival advantage for users of any NSAIDs in studies clearly defining non-use as less than once per week ( $\mathrm{pHR}=0.89(0.82-0.98))$.

Conclusions: Although this study did not show a clear association between analgesic use and ovarian cancer survival, further investigation with clearer definitions of use and information about post-diagnosis use is warranted.

Over 238000 women are diagnosed with ovarian cancer annually (International Agency for Research on Cancer, 2014) and 5-year survival is poor at $\sim 45 \%$ (Howlader et al, 2015). Identifying modifiable factors that could improve survival is therefore important. One possible factor is the use of analgesics such as nonsteroidal anti-inflammatory drugs (NSAIDs). Nonsteroidal anti-inflammatory drugs, including aspirin and non-aspirin
NSAIDs (NA-NSAIDs), inhibit the pro-inflammatory enzyme cyclooxygenase (COX). COX-2 is over-expressed in many cancers including ovarian (Maccio and Madeddu, 2012), and COXinhibition can reduce angiogenesis and trigger apoptosis (Xin et al, 2007). While improved survival among NSAID users has been reported for breast (Huang et al, 2015), prostate (Liu et al, 2014), and colorectal (Ye et al, 2014) cancers, two previous

*Correspondence: SC Dixon; E-mail: Suzanne.Dixon@qimrberghofer.edu.au

Received 7 August 2016; revised 9 December 2016; accepted 4 February 2017; published online 28 March 2017 
observational studies of ovarian cancer found no evidence that prediagnosis aspirin, NA-NSAID, or acetaminophen use was associated with survival (Minlikeeva et al, 2015; Nagle et al, 2015). However, both studies were underpowered to detect the likely modest effects. One trial reported no short-term (median followup 34 months) survival advantage among women with advanced ovarian cancer when a NA-NSAID was added to standard chemotherapy, but did not examine long-term outcomes (Reyners et al, 2012). Interestingly, a preliminary report (published as a conference abstract) has suggested that NSAID use postdiagnosis may be associated with improved survival (Poole et al, 2016). We used data from a large international consortium to examine the association between pre-diagnosis use of common analgesics and survival after a diagnosis of ovarian cancer. We hypothesised that NSAID users would experience a survival benefit compared to non-users.

\section{MATERIALS AND METHODS}

Study population. We pooled data from 12 case-control studies, which included 7694 women with invasive epithelial ovarian tumours, aged $<85$ years at diagnosis (Supplementary Table 1). Cancers of unknown behaviour ( $n=93$ high grade; $n=25$ low grade) were assumed to be invasive (Trabert et al, 2014). Most women (59\%) had serous cancers; $5 \%, 15 \%$, and $8 \%$ had mucinous, endometrioid, and clear cell cancers, respectively. Most cancers (63\%) had distant spread.

Exposure, outcome, and covariates. Studies provided data on self-reported pre-diagnosis analgesic use (Supplementary Table 2; harmonisation described previously (Trabert et al, 2014)). Two studies (UCI/UKO) did not report NA-NSAIDs data, so were only included in 'aspirin' and 'any NSAIDs' (aspirin plus non-aspirin) analyses. Regular use was defined as at least once per week $v s$ less often. Frequency, dose, and duration information was available for seven, three, and nine studies, respectively (Table 1). Studies provided data on vital status and time from diagnosis to death or end of follow-up. Disease recurrence/progression (from 3 studies) and cause of death (two studies) was known for $28 \%$ and $12 \%$ of women, respectively. Ethnicity, smoking status, education, body mass index (BMI), tumour stage, and residual disease was known for $99.8 \%, 89 \%, 87 \%, 92 \%, 99 \%$, and $24 \%$ of women, respectively.

Statistical analysis. Using Cox proportional hazards regression, we obtained hazard ratios (HRs) and 95\% confidence intervals (CIs) for the association between analgesic use and overall survival (OS) in each study. Potential confounders were selected a priori. We did not adjust for treatment type because treatment cannot influence pre-diagnosis analgesic use (as treatment occurs later) and these data were only available for $25 \%$ of the cohort. Main models were adjusted for age, education, and ethnicity. Survival time was left-truncated at recruitment to minimise potential bias from eligible women dying before they could be enroled. Following proportional hazards assumption checking (inspecting covariate associations with survival over time), we re-ran models including covariate ${ }^{\star}$ time interactions where these interactions were statistically significant (two studies). As the resulting estimates were virtually unchanged, final models did not include these interactions. Site-specific HRs for OS were combined using randomeffects meta-analysis. $I^{2}$ and $P$-values for heterogeneity (from chisquare tests) were inspected to assess inter-study heterogeneity. Associations between analgesic use and progression-free (PFS) and disease-specific survival (DSS) were estimated from single models, stratified by study, to maximise power. In the same manner we

Table 1. The association between regular pre-diagnosis use of common analgesic medications and overall survival following a diagnosis of invasive ovarian cancer

\begin{tabular}{|c|c|c|c|c|c|c|c|c|c|c|c|c|c|c|c|c|}
\hline \multirow[b]{2}{*}{ Exposure categorisation } & \multicolumn{4}{|c|}{ Aspirin } & \multicolumn{4}{|c|}{ Non-aspirin NSAIDs } & \multicolumn{4}{|c|}{ Any NSAIDs } & \multicolumn{4}{|c|}{ Acetaminophen } \\
\hline & $n$ & $\mathrm{pHR}^{\mathrm{a}}$ & $95 \% \mathrm{Cl}$ & $I^{2}$ & $n$ & $\mathrm{pHR}^{\mathrm{a}}$ & $95 \% \mathrm{Cl}$ & $I^{2}$ & $n$ & $\mathrm{pHR}^{\mathrm{a}}$ & $95 \% \mathrm{Cl}$ & $I^{2}$ & $n$ & $\mathrm{pHR}^{\mathrm{a}}$ & $95 \% \mathrm{Cl}$ & $1^{2}$ \\
\hline \multicolumn{17}{|l|}{ Regular use ${ }^{b}$} \\
\hline No & 6190 & Ref & & & 5196 & Ref & & & 4891 & Ref & & & 5908 & Ref & & \\
\hline Yes & 1286 & 0.96 & $0.88-1.04$ & 5.5 & 1570 & 0.97 & $0.89-1.05$ & 0.0 & 2563 & 0.94 & $0.86-1.03$ & 32.6 & 1264 & 1.01 & $0.93-1.10$ & 0.0 \\
\hline \multicolumn{17}{|l|}{ Frequency (7 studies) ${ }^{c}$} \\
\hline No regular use & 4268 & Ref & & & 3993 & Ref & & & 3297 & Ref & & & 4335 & Ref & & \\
\hline$<30$ days per month & 234 & 0.92 & $0.72-1.18$ & 31.0 & 498 & 0.93 & $0.79-1.09$ & 15.2 & 572 & 0.92 & $0.81-1.06$ & 0.0 & 532 & 1.05 & $0.85-1.30$ & $56.6^{d}$ \\
\hline Daily & 593 & 1.01 & $0.90-1.14$ & 0.0 & 768 & 0.99 & $0.89-1.10$ & 0.0 & 1226 & 0.98 & $0.89-1.07$ & 2.4 & 419 & 0.99 & $0.86-1.13$ & 0.0 \\
\hline \multicolumn{17}{|l|}{ Daily dose (3 studies) } \\
\hline No regular use & 1330 & Ref & & & 1244 & Ref & & & 899 & Ref & & & 1484 & Ref & & \\
\hline Low & 125 & 0.90 & $0.58-1.39$ & 51.0 & 253 & 0.98 & $0.81-1.18$ & 0.0 & 299 & 0.95 & $0.79-1.13$ & 0.0 & 65 & 0.90 & $0.63-1.27$ & 0.0 \\
\hline High & 205 & 0.92 & $0.69-1.22$ & 37.2 & 230 & 1.10 & $0.91-1.33$ & 0.0 & 396 & 0.92 & $0.69-1.24$ & 64.2 & 289 & 1.09 & $0.89-1.34$ & 18.5 \\
\hline \multicolumn{17}{|l|}{ Duration (9 studies) f $^{f}$} \\
\hline No regular use & 3919 & Ref & & & 3523 & Ref & & & 2889 & Ref & & & 4201 & Ref & & \\
\hline$<60$ months & 426 & 0.96 & $0.82-1.13$ & 22.1 & 483 & 0.99 & $0.87-1.13$ & 0.0 & 689 & 0.94 & $0.80-1.11$ & 40.9 & 253 & 1.04 & $0.87-1.24$ & 0.0 \\
\hline $60+$ months & 559 & 1.01 & $0.89-1.14$ & 0.0 & 519 & 0.90 & $0.78-1.04$ & 0.0 & 930 & 0.98 & $0.86-1.13$ & 29.8 & 460 & 1.00 & $0.83-1.21$ & 34.4 \\
\hline
\end{tabular}

Abbreviations: $\mathrm{Cl}=$ confidence interval; $\mathrm{NSAID}=$ nonsteroidal anti-inflammatory drug; $\mathrm{pHR}=$ pooled hazard ratio.

${ }^{a}$ Adjusted for age (in years), ethnicity (if $<95 \%$ of participants were of the same ethnicity) (White/Hispanic/Black/Asian/Other), and education (Less than high-school/Completed high-school including some college/College graduate/Education status unknown).

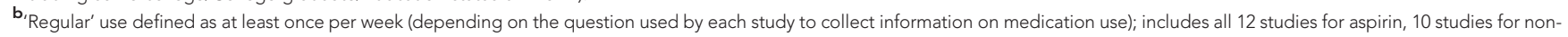
aspirin NSAIDs (excluding UCI/UKO, which did not report these data), 12 studies for any NSAIDs (aspirin or non-aspirin), and 11 studies (excluding UKO) for acetaminophen

${ }^{c}$ Frequency analyses were conducted in 7 studies with available data (AUS, DOV, HAW, HOP, MAL, NCO, and USC). Frequency of daily use of any NSAID may be slightly underestimated (while less than daily use may be overestimated), because if aspirin and non-aspirin NSAIDs were each taken $<7$ days per week, this is categorised as less than daily use of any NSAID although it is possible that at least one type of NSAID was taken 7 days per week (this cannot be determined from the data available).

${ }^{\text {d }}$ Statistically significant heterogeneity in the pHR across studies.

e Low/High defined as $</ \geq 100 \mathrm{mg} /$ day for aspirin, and $</ \geq 500 \mathrm{mg} /$ day for non-aspirin NSAIDs and acetaminophen; includes 3 studies with available data (HAW, HOP, and NCO).

fDuration analyses were conducted in 9 studies with available data (CON, DOV, HAW, HOP, MAL, NEC, NJO, UCl, and USC) for aspirin and acetaminophen, and in 8 studies (excluding UCl) for non-aspirin NSAIDs and any NSAIDs. 
conducted analyses stratified by characteristics likely to modify the association (age, BMI, and disease stage).

This analysis and each contributing study received approval from the appropriate institutional review board/ethics committee. All participants provided written informed consent.

\section{RESULTS}

Population characteristics. Mean age at diagnosis was 58 years, $88 \%$ of women were non-Hispanic white, and $27 \%$ were tertiaryeducated. Of the 7694 women, $17 \%, 23 \%$, and $18 \%$ had regularly used aspirin, NA-NSAIDs, and acetaminophen, respectively (Supplementary Table 1). Median follow-up (using reverse Kaplan-Meier (Schemper and Smith, 1996)) was 8.0 years. Over half the women $(n=4273,56 \%)$ died and 5 -year survival was $55 \%$ (Supplementary Table 1), yielding $90 \%$ power to detect a $\mathrm{HR}=0.90$ for NSAID users. Among studies with progression/ cause-of-death information, $73 \%$ of women experienced progression and $95 \%$ of deaths were from ovarian cancer.

Primary results. Regular use of analgesics was not associated with OS (pHRs (95\% CI): aspirin $0.96(0.88-1.04)$; NA-NSAIDs 0.97 (0.89-1.05); any NSAIDs 0.94 (0.86-1.03); acetaminophen 1.01
(0.93-1.10)), nor were frequency, dose, or duration of use (Table 1; Figure 1). There was no significant inter-study heterogeneity (Figure 1). Additional adjustment for tumour stage and grade, residual disease, BMI and smoking status did not appreciably alter effect estimates. Cross-classifying frequency by dosage (among five studies with data) did not demonstrate consistent associations, and long-term ( $\geq 5$ years) daily use was not associated with survival. Truncating follow-up at five years (when most deaths would be cancer-related) did not affect results. No significant associations were observed with PFS (any NSAIDs, HR $=0.96 ; 95 \%$ CI $0.80-1.14)$ or DSS $(\mathrm{HR}=0.98 ; 95 \%$ CI $0.82-1.17)$. There was no significant variation by tumour histology (Table 2; $P$-interaction $=0.3-0.7)$. Excluding the two studies which had previously examined this association (Minlikeeva et al, 2015; Nagle et al, 2015) (whose participants comprised 25\% of this analysis), two studies with high survival rates (these studies recruited a number of prevalent cases), or two studies who asked only about recent use (past 5 years), did not substantially alter effect estimates.

To minimise exposure misclassification due to heterogeneous questions between studies, we repeated analyses restricted to six studies clearly defining non-use as less than once per week (Supplementary Table 3). This showed a significant survival advantage among regular users of any NSAIDs $(\mathrm{pHR}=0.89 ; 95 \%$

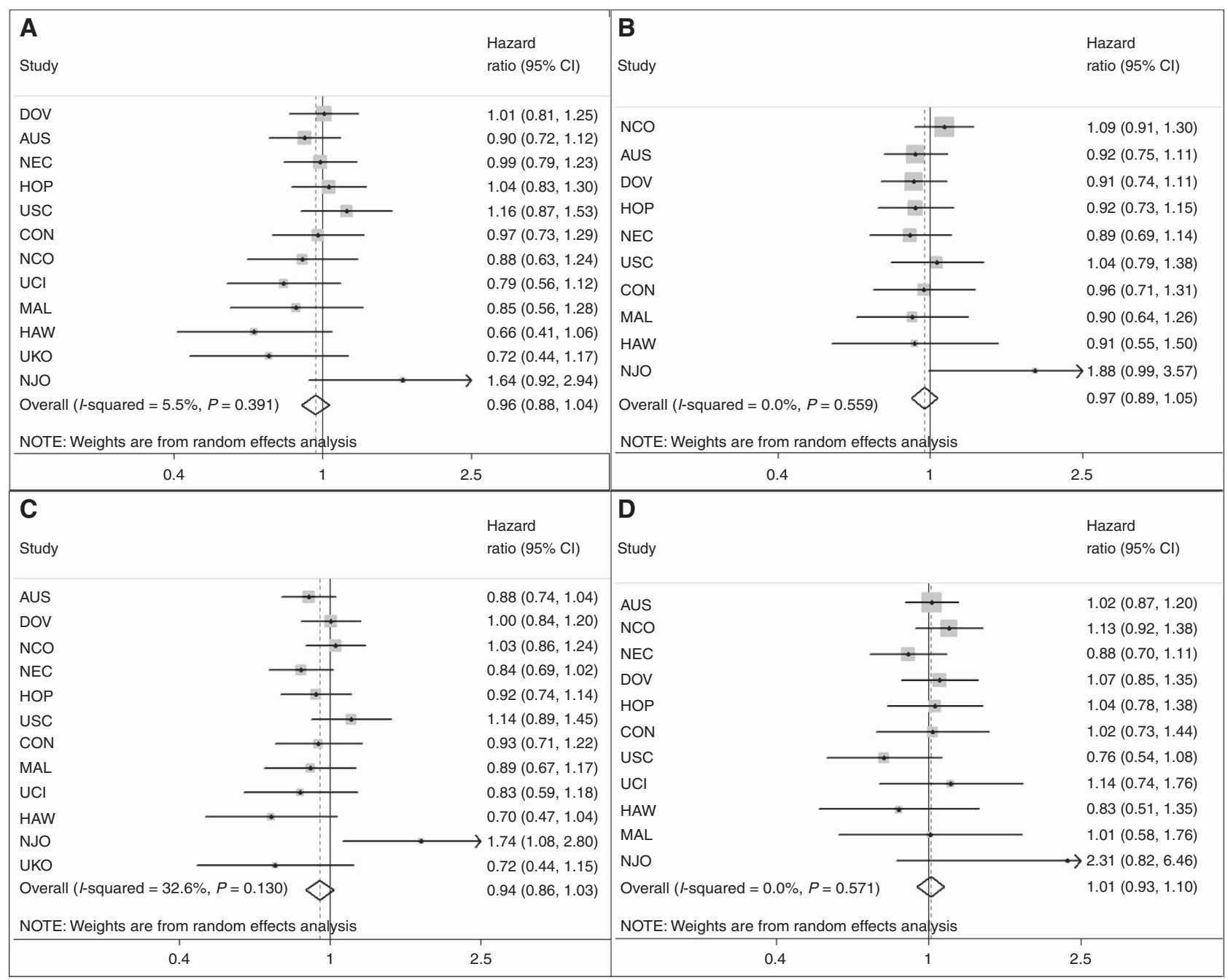

Figure 1. The association between regular pre-diagnosis use of common analgesic medications and overall survival following a diagnosis of invasive ovarian cancer, adjusted for age, ethnicity (if < $95 \%$ of participants are of the same ethnicity), and education. (A) Aspirin, (B) non-aspirin NSAIDs, (C) any NSAIDs, (D) acetaminophen. 
Table 2. The association between regular pre-diagnosis use of common analgesic medications and overall survival following a diagnosis of invasive ovarian cancer, by histologic subtype

\begin{tabular}{|c|c|c|c|c|c|c|c|c|c|c|c|c|c|c|c|c|}
\hline \multirow[b]{2}{*}{ Histologic Subtype } & \multicolumn{4}{|c|}{$\begin{array}{l}\text { Aspirin (Regular } \\
\text { vs no regular use) } \\
\text { (12 studies) }\end{array}$} & \multicolumn{4}{|c|}{$\begin{array}{c}\text { Non-aspirin NSAIDs } \\
\text { (Regular }^{a} \text { vs no regular use) } \\
\text { (10 studies) }\end{array}$} & \multicolumn{4}{|c|}{$\begin{array}{l}\text { Any NSAIDs (Regular } \\
\text { vs no regular use) } \\
\text { (12 studies) }\end{array}$} & \multicolumn{4}{|c|}{$\begin{array}{c}\text { Acetaminophen (Regular } \\
\text { vs no regular use) } \\
\text { (11 studies) }\end{array}$} \\
\hline & $n^{b}$ & $\mathrm{pHR}^{\mathrm{c}}$ & $95 \% \mathrm{Cl}$ & $I^{2}$ & $n^{b}$ & $\mathrm{pHR}^{\mathrm{c}}$ & $95 \% \mathrm{Cl}$ & $I^{2}$ & $n^{b}$ & $\mathrm{pHR}^{\mathrm{c}}$ & $95 \% \mathrm{Cl}$ & $I^{2}$ & $n^{b}$ & $\mathrm{pHR}^{\mathrm{c}}$ & $95 \% \mathrm{Cl}$ & $I^{2}$ \\
\hline Serous $^{\mathrm{d}}$ & 4386 & .04 & $92-1.17$ & 24.0 & 4034 & 1.01 & & 28.9 & 4394 & & & 2.7 & 4255 & & & 1.3 \\
\hline grade & 4065 & 02 & & 11.0 & 3728 & & & 30.6 & 4067 & & & 9.5 & & & & 1.8 \\
\hline & 265 & 1.71 & $0.76-3.87$ & 31.2 & 252 & 1.28 & & 46.9 & 277 & & & 25.6 & 246 & & & 34.6 \\
\hline Mucinous & 248 & 0.92 & $.42-2.01$ & 0.0 & 287 & 1.50 & $0.80-2.80$ & 0.0 & 353 & & & 0.0 & 209 & & & 34.2 \\
\hline Endometrioid & 1126 & 0.87 & $0.63-1.21$ & 0.0 & 1000 & 0.93 & $0.69-1.27$ & 0.0 & 1118 & 0.94 & 0.66 & 37.5 & 982 & 0.96 & 1.53 & 29.2 \\
\hline Clear cell & 474 & 1.22 & $0.74-2.02$ & 14.2 & 452 & 0.87 & $0.50-1.53$ & 36.2 & 548 & 1.01 & $0.61-1.67$ & 43.6 & 392 & 0.93 & $0.37-2.33$ & $61.6^{\mathrm{e}}$ \\
\hline \multicolumn{17}{|c|}{ 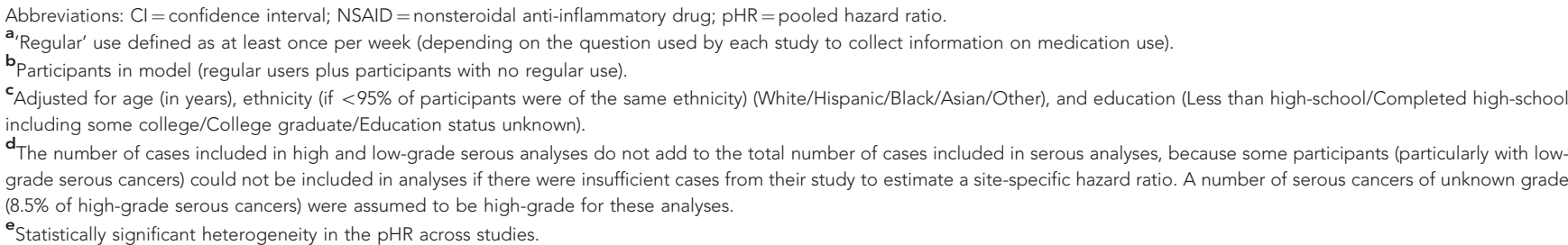 } \\
\hline
\end{tabular}

CI 0.82-0.98). When we excluded studies with low exposure prevalence $(<10 \%)$, a similar association was seen among the eight remaining studies (including the six above; any NSAIDs $\mathrm{HR}=0.92$; 95\% CI 0.85-0.99).

In stratified analyses, an inverse association between any NSAID use and survival was seen among women aged $\geq 60$ at diagnosis $(\mathrm{pHR}=0.90$; 95\% CI $0.82-0.99)$ or with BMI $<25 \mathrm{~kg} \mathrm{~m}^{-2}(0.86$; $0.77-0.95)$. A non-significant inverse association was seen among women with early-stage (localised/regional) tumours $(\mathrm{pHR}=0.87$; 95\% CI 0.74-1.04; Supplementary Table 4).

\section{DISCUSSION}

Overall, we did not find convincing evidence to support an association between use of aspirin, NA-NSAIDs, or acetaminophen prior to diagnosis and ovarian cancer survival. Although most HRs for aspirin and NA-NSAIDs were $<1.0$, none was statistically significant. Our results are consistent with the two previous observational studies (Minlikeeva et al, 2015; Nagle et al, 2015) examining pre-diagnosis use of NSAIDs. The relationship did not vary by histologic subtype.

Questions used to define regular use differed between studies. When we restricted analyses to a subset of studies clearly defining non-use as less than once per week, use of any NSAIDs was associated with significantly improved survival. No notable characteristics differentiated these studies from others in the main analyses. This may suggest that exposure misclassification attenuated our primary results, and that analyses using a more consistent definition of use might have more power to detect modest associations with survival. However the results of these post hoc analyses require validation and should be interpreted with caution. The apparent lack of association for long-term daily use of any NSAIDs among the five studies with this information (this included studies with potential misclassification of non-users) could reflect the fact that daily aspirin users are more likely to use a low-dose preparation, which may be insufficient to confer a survival benefit.

We did not have information on medication use after diagnosis, which may be a more relevant time-window, especially if women change their use after diagnosis. A recent meta-analysis of NSAID use and breast cancer survival found post- but not pre-diagnosis use was associated with improved survival (Huang et al, 2015), a pattern also suggested by the preliminary report (conference abstract) examining post-diagnosis use among a small number $(N=602)$ of ovarian cancer patients (Poole et al, 2016). Unless change in use is associated with survival, the likely effect of using pre-diagnosis data to estimate post-diagnosis use would be random misclassification, attenuating any real association. We had insufficient data to stratify by post-diagnosis prognostic factors such as treatment received, but additional adjustment for stage/ grade of disease (which predict treatment type) and amount of residual disease after surgery did not appreciably alter our estimates.

In conclusion, we did not find convincing evidence of an association between pre-diagnosis analgesic use and ovarian cancer survival. However, the modest associations in subgroup analyses suggest we cannot exclude the possibility that NSAID use is associated with survival (but we could not detect this due to exposure misclassification and/or a sub-optimal exposure window). Further investigation with more consistent definitions of analgesic use/non-use (including by selective/non-selective COX-2 inhibition) and information about post-diagnosis use is warranted.

\section{ACKNOWLEDGEMENTS}

This work was supported by: the National Cancer Institute at the U.S. National Institutes of Health (K07-CA095666, K07-CA80668, K22-CA138563, N01-CN025403, N01-CN55424, N01-PC67001, N01-PC67010, P01-CA17054, P30-CA072720, P30-CA14089, P50-CA105009, P50-CA159981, R01-CA058860, R01-CA074850, R01-CA080742, R01-CA092044, R01-CA095023, R01-CA54419, R01-CA58598, R01-CA61107, R01-CA61132, R01-CA76016, R01CA87538, R01-CA112523, R01-CA126841, R03-CA113148, and R03-CA115195); the U.S. Army Medical Research and Materiel Command (DAMD17-01-1-0729, DAMD17-02-1-0666, DAMD 17-02-1-0669, and W81XWH-10-1-0280); the National Health and Medical Research Council of Australia (199600, 400281, 1073898 and P.M.W. fellowship); Cancer Councils of Queensland, Victoria, New South Wales, South Australia and Tasmania and the Cancer Foundation of Western Australia (Multi-State Grant Applications 
191, 211, and 182); the Danish Cancer Society (94-222-52); the Mermaid I project; the Cancer Institute of New Jersey; the U.K. National Institute for Health Research University College London Hospital Biomedical Research Centre; the U.S. Public Health Service (PSA-042205); the Lon V. Smith Foundation (LVS-39420); The Eve Appeal (The Oak Foundation); and the California Cancer Research Program (00-01389V-20170 and 2II0200). We thank all the individuals who took part in this study and all the researchers, clinicians, and technical and administrative staff who have made possible the many studies contributing to this work. In particular, for their contribution to the design and conduct of the individual studies that contributed to the analysis, we thank: D. Bowtell, G. Chenevix-Trench, D. Gertig, A. Green, P. Parsons, N. Hayward and D. Whiteman (AUS); I. Orlow and L. Rodriguez-Rodriguez (NJO); and I. Jacobs, M.Widschwendter, E. Wozniak, A. Ryan, J. Ford, N. Balogun and C. Karpinskyj (UKO). We also thank the intramural research program of the US National Cancer Institute for harmonisation of the analgesic variables.

\section{CONFLICT OF INTEREST}

The authors declare no conflict of interest.

\section{REFERENCES}

Howlader N, Noone AM, Krapcho M, Garshell J, Miller D, Altekruse S, Kosary CL, Yu M, Ruhl J, Tatalovich Z, Mariotto A, Lewis DR, Chen HS, Feuer EJ, Cronin KA (2015) SEER Cancer Statistics Review, 1975-2012. National Cancer Institute: Bethesda, MD, USA.

Huang X-z, Gao P, Sun J-x, Song Y-x, Tsai C-c, Liu J, Chen X-w, Chen P, Xu H-m, Wang Z-n (2015) Aspirin and nonsteroidal anti-inflammatory drugs after but not before diagnosis are associated with improved breast cancer survival: a meta-analysis. Cancer Causes Control 26: 589-600.

International Agency for Research on Cancer (2014) GLOBOCAN 2012. Available at http://globocan.iarc.fr/.

Liu Y, Chen J-Q, Xie L, Wang J, Li T, He Y, Gao Y, Qin X, Li S (2014) Effect of aspirin and other non-steroidal anti-inflammatory drugs on prostate cancer incidence and mortality: a systematic review and meta-analysis. BMC Med 12: 55-55.

Maccio A, Madeddu C (2012) Inflammation and ovarian cancer. Cytokine 58: 133-147.

Minlikeeva AN, Freudenheim JL, Lo-Ciganic W-H, Eng KH, Friel G, Diergaarde B, Modugno F, Cannioto R, Gower E, Szender JB, Grzankowski K, Odunsi K, Ness RB, Moysich KB (2015) Use of common analgesics is not associated with ovarian cancer survival. Cancer Epidemiol Biomarkers Prev 24: 1291-1294.

Nagle CM, Ibiebele TI, DeFazio A, Protani MM, Webb PM (2015) Aspirin, nonaspirin nonsteroidal anti-inflammatory drugs, acetaminophen and ovarian cancer survival. Cancer Epidemiol 39: 196-199.

Poole EM, Rice MS, Tworoger SS (2016) Abstract B29: Pre- and postdiagnosis analgesic use and ovarian cancer survival. Clin Cancer Res 22: B29.

Reyners AKL, de Munck L, Erdkamp FLG, Smit WM, Hoekman K, Lalisang RI, de Graaf H, Wymenga ANM, Polee M, Hollema H, van Vugt M, Schaapveld M, Willemse PHB. DoCaCel Study G (2012) A randomized phase II study investigating the addition of the specific COX-2 inhibitor celecoxib to docetaxel plus carboplatin as first-line chemotherapy for stage IC to IV epithelial ovarian cancer, Fallopian tube or primary peritoneal carcinomas: the DoCaCel study. Ann Oncol 23: 2896-2902.

Schemper M, Smith TL (1996) A note on quantifying follow-up in studies of failure time. Control Clin Trials 17: 343-346.

Trabert B, Ness RB, Lo-Ciganic WH, Murphy MA, Goode EL, Poole EM, Brinton LA, Webb PM, Nagle CM, Jordan SJ, Risch HA, Rossing MA, Doherty JA, Goodman MT, Lurie G, Kjaer SK, Hogdall E, Jensen A, Cramer DW, Terry KL, Vitonis A, Bandera EV, Olson S, King MG, Chandran U, Anton-Culver H, Ziogas A, Menon U, Gayther SA, Ramus SJ, Gentry-Maharaj A, Wu AH, Pearce CL, Pike MC, Berchuck A, Schildkraut JM, Wentzensen N (2014) Aspirin, nonaspirin nonsteroidal anti-inflammatory drug, and acetaminophen use and risk of invasive epithelial ovarian cancer: a pooled analysis in the Ovarian Cancer Association Consortium. J Natl Cancer Inst 106: djt431.

Xin B, Yokoyama Y, Shigeto T, Mizunuma H (2007) Anti-tumor effect of nonsteroidal anti-inflammatory drugs on human ovarian cancers. Pathol Oncol Res 13: 365-369.

Ye XF, Wang J, Shi WT, He J (2014) Relationship between aspirin use after diagnosis of colorectal cancer and patient survival: a meta-analysis of observational studies. Br J Cancer 111: 2172-2179.

${ }^{1}$ Gynaecological Cancers Group, QIMR Berghofer Medical Research Institute, 300 Herston Road, Brisbane, Queensland 4006, Australia; ${ }^{2}$ The University of Queensland, School of Public Health, Level 2 Public Health Building (887), Corner of Herston Road \& Wyndham Street, Brisbane, Queensland 4006, Australia; ${ }^{3}$ Division of Cancer Epidemiology and Genetics, National Cancer Institute, 9609 Medical Center Drive, Bethesda, MD 20892-9774, USA; ${ }^{4}$ Vanderbilt Epidemiology Center, Vanderbilt University School of Medicine, 2525 West End Avenue, Nashville, TN 37203, USA; ${ }^{5}$ Department of Community and Family Medicine, Duke University Medical Center, 2424 Erwin Road, Suite 602, Durham, NC 27710, USA; ${ }^{6}$ Cancer Control and Population Sciences, Duke Cancer Institute, DUMC Box 3917, 10 Bryan Searle Drive, Seeley Mudd Building, 2nd floor, Durham, NC 27710, USA; ${ }^{7}$ Department of Cancer Prevention and Control, Roswell Park Cancer Institute, Elm and Carlton Streets, Buffalo, NY 14263, USA; ${ }^{8}$ Centre for Cancer Research, the Westmead Institute for Medical Research, The University of Sydney, 176 Hawkesbury Road, Sydney, NSW 2145, Australia; ${ }^{9}$ Department of Gynaecological Oncology, Westmead Hospital, Cnr Hawkesbury Road and Darcy Road, Sydney, New South Wales 2145, Australia; ${ }^{10}$ Cancer Genetics and Genomics Laboratory, Peter MacCallum Cancer Centre, St Andrews Place, Melbourne, Victoria 3002, Australia; ${ }^{11}$ Department of Chronic Disease Epidemiology, Yale School of Public Health, LEPH 413, 60 College Street, New Haven, CT 06510, USA; ${ }^{12}$ Program in Epidemiology, Division of Public Health Sciences, Fred Hutchinson Cancer Research Center, 1100 Fairview Avenue North, Seattle, WA 98109-1024, USA; ${ }^{13}$ Department of Epidemiology, University of Washington, 1959 NE Pacific Street, Health Sciences Bldg, F-262, Seattle, WA 98195, USA; ${ }^{14}$ Department of Epidemiology, The Geisel School of Medicine at Dartmouth, 1 Medical Center Drive, 7927 Rubin Building, Lebanon, NH 03756, USA; ${ }^{15}$ Cancer Prevention and Control, Samuel Oschin Comprehensive Cancer Institute, Cedars-Sinai Medical Center, 8700 Beverly Blvd., Los Angeles, CA 90048, USA; ${ }^{16}$ Community and Population Health Research Institute, Department of Biomedical Sciences, Cedars-Sinai Medical Center, 8700 Beverly Blvd., Los Angeles, CA 90048, USA; ${ }^{17}$ Division of Gynecologic Oncology, Department of Obstetrics, Gynecology and Reproductive Sciences, University of Pittsburgh School of Medicine, 300 Halket Street, Pittsburgh, PA 15213, USA; ${ }^{18}$ Ovarian Cancer Center of Excellence, Women's Cancer Research Program, Magee-Women's Research Institute and University of Pittsburgh Cancer Institute, 204 Craft Avenue, Pittsburgh, PA 15213, USA; ${ }^{19}$ Department of Epidemiology, University of Pittsburgh Graduate School of Public Health, 130 De Soto Street, Pittsburgh, PA 15261, USA; ${ }^{20}$ The $^{2}$ University of Texas School of Public Health, 1200 Herman Pressler, Suite W130, Houston, TX 77030, USA; ${ }^{21}$ Department of Virus, 
Lifestyle and Genes, Danish Cancer Society Research Center, Strandboulevarden 49, Copenhagen Ø DK-2100, Denmark; ${ }^{22}$ Department of Gynaecology, Rigshospitalet, University of Copenhagen, Blegdamsvej 9, Copenhagen $\varnothing$ DK-2100, Denmark; ${ }^{23}$ Molecular Unit, Department of Pathology, Herlev Hospital, University of Copenhagen, Herlev Ringvej 75, Herlev DK-2370, Denmark; ${ }^{24}$ Department of Obstetrics and Gynecology, Duke University Medical Center, 25171 Morris Bldg, Durham, NC 27710, USA; ${ }^{25}$ Obstetrics and Gynecology Epidemiology Center, Brigham and Women's Hospital, 221 Longwood Avenue, Richardson Fuller Building, Boston, MA 02115, USA; ${ }^{26}$ Department of Epidemiology, Harvard T.H. Chan School of Public Health, 677 Huntington Avenue, Boston, MA 02115, USA; ${ }^{27}$ Channing Division of Network Medicine, Brigham and Women's Hospital and Harvard Medical School, 181 Longwood Avenue, Boston, MA 02115, USA; ${ }^{28}$ Cancer Prevention and Control Program, Rutgers Cancer Institute of New Jersey, 195 Little Albany Street, New Brunswick, NJ 08903, USA; ${ }^{29}$ Rutgers School of Public Health, 683 Hoes Lane West, Piscataway, NJ 08854, USA; ${ }^{30}$ Cancer Surveillance Research Program, Rutgers Cancer Institute of New Jersey, 195 Little Albany Street, New Brunswick, NJ 08903, USA; ${ }^{31}$ Department of Epidemiology, University of California Irvine, 224 Irvine Hall, Irvine, CA 92697-7550, USA; ${ }^{32}$ Genetic Epidemiology Research Institute, UCl Center for Cancer Genetics Research \& Prevention, School of Medicine, University of California Irvine, 224 Irvine Hall, Irvine, CA 92697-7550, USA; ${ }^{33}$ Women's Cancer, Institute for Women's Health, University College London, Maple House 1st Floor, 149 Tottenham Court Road, London W1T 7DN, UK; ${ }^{34}$ Department of Preventive Medicine, Keck School of Medicine, University of Southern California Norris Comprehensive Cancer Center, 1441 Eastlake Avenue, Los Angeles, CA 90033, USA; ${ }^{35}$ School of Women's and Children's Health, University of New South Wales, Level 1, Women's Health Institute, Royal Hospital for Women, Barker Street, Randwick, New South Wales 2031, Australia; ${ }^{36}$ The Kinghorn Cancer Centre, Garvan Institute of Medical Research, 384 Victoria Street, Darlinghurst, New South Wales 2010, Australia; ${ }^{37}$ Department of Epidemiology, University of Michigan School of Public Health, 1415 Washington Heights, SPH Tower, Ann Arbor, MI 48109-2029, USA and ${ }^{38}$ Department of Epidemiology and Biostatistics, Memorial Sloan Kettering Cancer Center, 307 East 63rd Street, New York, NY 10065, USA

Supplementary Information accompanies this paper on British Journal of Cancer website (http://www.nature.com/bjc) 